\title{
Making Use of Plant uORFs to Control Transgene Translation in Response to Pathogen Attack
}

\author{
Gan Ai, ${ }^{1}$ Jin Liu, ${ }^{1}$ Xiaowei Fu, ${ }^{1}$ Tianli Li, ${ }^{1}$ Hai Zhu, ${ }^{1}$ Ying Zhai ${ }^{D},{ }^{2}$ Chuyan Xia $\mathbb{D}^{1},{ }^{1}$ \\ Weiye Pan, ${ }^{1}$ Jialu Li, ${ }^{1}$ Maofeng Jing $\mathbb{D}^{1},{ }^{1}$ Danyu Shen, ${ }^{1}$ Ai Xia, ${ }^{1}$ and Daolong Dou $\mathbb{D}^{1}$ \\ ${ }^{1}$ College of Plant Protection, Academy for Advanced Interdisciplinary Studies, Nanjing Agricultural University, \\ Nanjing 210095, China \\ ${ }^{2}$ Department of Plant Pathology, Washington State University, Pullman, WA 99164, USA
}

Correspondence should be addressed to Daolong Dou; ddou@njau.edu.cn

Gan Ai and Jin Liu contributed equally to this work.

Received 29 October 2021; Accepted 6 January 2022; Published 3 February 2022

Copyright (c) 2022 Gan Ai et al. Exclusive Licensee Nanjing Agricultural University. Distributed under a Creative Commons Attribution License (CC BY 4.0).

\begin{abstract}
Reducing crop loss to diseases is urgently needed to meet increasing food production challenges caused by the expanding world population and the negative impact of climate change on crop productivity. Disease-resistant crops can be created by expressing endogenous or exogenous genes of interest through transgenic technology. Nevertheless, enhanced resistance by overexpressing resistance-produced genes often results in adverse developmental affects. Upstream open reading frames (uORFs) are translational control elements located in the $5^{\prime}$ untranslated region (UTR) of eukaryotic mRNAs and may repress the translation of downstream genes. To investigate the function of three uORFs from the $5^{\prime}$-UTR of ACCELERATED CELL 11 $\left(\mathrm{uORFs}_{\mathrm{ACD} 11}\right.$ ), we develop a fluorescent reporter system and find $\mathrm{uORFs}_{\mathrm{ACD} 11}$ function in repressing downstream gene translation. Individual or simultaneous mutations of the three $\mathrm{uORFs}_{\mathrm{ACD} 11}$ lead to repression of downstream translation efficiency at different levels. Importantly, $\mathrm{uORFs}_{\mathrm{ACD} 11}$-mediated translational inhibition is impaired upon recognition of pathogen attack of plant leaves. When coupled with the PATHOGENESIS-RELATED GENE 1 (PR1) promoter, the uORFs $_{\text {ACD11 }}$ cassettes can upregulate accumulation of Arabidopsis thaliana LECTIN RECEPTOR KINASE-VI.2 (AtLecRKVI.2) during pathogen attack and enhance plant resistance to Phytophthora capsici. These findings indicate that the $\mathrm{uORFs}_{\mathrm{ACD11}}$ cassettes can be a useful toolkit that enables a high level of protein expression during pathogen attack, while for ensuring lower levels of protein expression at normal conditions.
\end{abstract}

\section{Introduction}

Food production demands increase with the expanding world population and the negative impact of global climate change [1-3]. However, crop diseases become a major challenge to modern agriculture [4], with about $15 \%$ and $3 \%$ yield reduction caused by fungal/bacterial and viral pathogens, respectively [5, 6]. Microbial infection is a more severe threat to certain crops such as potato, in which it causes nearly $30 \%$ yield loss $[5,6]$. The integration of enhanced resistance into new crop varieties by conventional breeding requires selection of desirable traits over several generations [7]. In contrast, ectopic expression of resistance-conferring genes is a rapid and powerful approach for enhancing crop disease resistance $[4,8]$.
The genetic engineering approach relies on our expanding knowledge of plant immune mechanisms [9]. There are successful examples of specifically enhancing plant resistance to certain pathogens via ectopic expression of corresponding immunity-related genes, such as the legume-like lectin receptor kinase LecRK-I.9 which is recognizing RXLR effector protein IPI-O [10] and the bacterial Elongation Factor Thermo Unstable (EF-Tu) Receptor (EFR) [11, 12]. However, overexpressing resistance-conferring genes often lead to deleterious pleiotropic effects that antagonize normal plant growth [13-15]. For example, overexpression of Arabidopsis thaliana NONEXPRESSER OF PR GENES 1 (AtNPR1) enhances plant disease resistance with conditional side effects [16-18]. Thus, developing novel strategies 
to fine-tune transgene expression and translation is critical for balancing the trade-off between plant growth and the improved defense. A promising approach is the adoption of pathogen-inducible promoters.

Genes driven by pathogen-inducible promoters are specifically induced upon pathogen infection. Thus, pathogeninducible promoter-controlled expression of immunityrelated genes may be a rational solution to reduce unnecessary growth inhibition [19-21]. For example, the promotor of Glycine max polyphenol oxidase gene, GmPPO12, is a pathogen-induced promoter that could be used in transgenic engineering [22]. However, pathogen-inducible promoters often auto-activate transgenes in plants [23]. For example, transgenic tobacco plants expressing the cryptogein or popA elicitor driven by the pathogen-inducible promoter $h s r 203 J$ show broad-spectrum resistance, but some lines display runaway cell death due to hsr203J-induced gene auto-activation $[24,25]$. Thus, regulatory elements with minimal side effects should be identified and used for fine-tuning transgene products at transcriptional and/or translational levels. A group of such candidates are from the translational control elements named upstream open reading frames (uORFs).

uORFs lie in the $5^{\prime}$ untranslated region (UTR) of eukaryotic mRNAs. They usually repress the translation of main open reading frames (mORFs), which are located downstream of uORFs [26, 27]. In Arabidopsis, there are 10,104 annotated uORFs found in about $37 \%$ of the total mRNAs [28, 29]. There are 8,531 out of $13,297(64 \%)$ uORF-containing mRNAs harboring two or more uORFs according to the uORFlight database [30], indicating the prevalence of uORF-regulated downstream gene expression. Recent studies confirm the important roles of uORFs in regulating plant growth and defense [31-33]. In addition, uORFs have been successfully used in engineering plant immunity. For example, transgenic rice expressing AtNPR1 driven by Arabidopsis TL1-BINDING TRANSCRIPTION FACTOR 1 (AtTBF1) uORFs (uORFs AtTBF1 $_{\text {( }}$ ) exhibits enhanced broad-spectrum disease resistance with no apparent growth retardation [34].

ACD11 is a ceramide-1-phosphate transfer protein that negatively regulates plant immunity $[35,36]$. The protein stability of ACD11 is regulated by the E3 ligase XBA35.2 [37]. We previously also showed that ACD11 can be stabilized via its physical interactions with BINDING PARTNER OF ACD11 1 (BPA1)-like (BPL) family proteins [36]. However, whether there are other mechanisms regulating ACD11 gene products is still unknown. AtLecRK-VI.2 harbors an extracellular lectin motif $[38,39]$ and positively regulates Arabidopsis resistance to bacterial pathogens [40]. LecRKVI.2 also functions as a key component of systemic acquired resistance (SAR) by recognizing the putative SAR mobile signal extracellular nicotinamide adenine dinucleotide (eNAD+) [41]. Heterologous expression of LecRK-VI.2 in $N$. benthamiana increases plant resistance to a broad range of bacteria pathogens [42].

To identify uORF's capacity of regulating downstream gene products, we have developed a fluorescence-based method to evaluate how a uORF might regulate protein production. Three predicted uORFs from the $5^{\prime}$ UTR of ACD 11 were evaluated using this system. Individual or simultaneous mutations of the three $\mathrm{uORFs}_{\mathrm{ACD} 11}$ lead to repression of downstream translation variously. This translational inhibition was further impaired by pathogen inoculation. Therefore, we combined the $\mathrm{UORFs}_{\mathrm{ACD} 11}$ cassettes with the PATHOGENESIS-RELATED GENE 1 (PR1) promoter and demonstrated that they can fine-tune AtLecRK-VI.2-mediated resistance in transgenic $N$. benthamiana plants, indicating that the $\mathrm{uORFs}_{\mathrm{ACD} 11}$ cassettes can be a useful toolkit for engineering crop disease resistance with desired fitness cost.

\section{Materials and Methods}

2.1. Plasmid Constructs. To build fluorescence- and luminescence-based reporter system, full length of GFP coding sequence (CDS), terminator of nopaline synthase gene (tNOS), CaMV 35S promoter (35S), and Luciferase CDS were amplified and inserted sequentially into the pSuper vector which contains a MAS promoter. $5^{\prime}-\mathrm{UTR}_{\mathrm{TBF} 1}, 5^{\prime}$ $\mathrm{UTR}_{\mathrm{ACD} 11}$, and AtLecRK-VI.2 with a C-terminal-fused FLAG tag were amplified from WT Arabidopsis (Col-0). Site-directed mutagenesis of $\mathrm{uORFs}_{\mathrm{ACD} 11}$ was performed to change ATG to CTG. To generate transgenic Arabidopsis expressing NAT-GFP, $5^{\prime} \mathrm{UTR}_{\mathrm{ACD} 11}$, GFP, and tNOS were inserted sequentially into pSuper. To generate transgenic $N$. benthamiana, different combinations of $\mathrm{uORFs}_{\mathrm{ACD} 11}$ cassettes and AtLecRK-VI.2 with C-terminal fused FLAG tag and tNOS were inserted sequentially into pSuper. Primers used in this study are listed in Table S1.

\subsection{Plant Materials and Transgenic Plants. N. benthamiana} plants used in this study were grown in the glasshouse at $26^{\circ} \mathrm{C}$ under a 16-hours light/8-hours dark photoperiod for 6 weeks. Arabidopsis were grown at $24^{\circ} \mathrm{C}$ under a 12 -hours light/12-hours dark photoperiod and $60 \%$ relative humidity for one month.

To generate transgenic Arabidopsis plants, the indicated plasmid was introduced into Agrobacterium strain GV3101. Arabidopsis WT (Col-0) plants were transformed using the standard Agrobacterium-mediated floral dip protocol. Transgenic plants were screened on 1/2 MS medium containing $50 \mathrm{mg} / \mathrm{l}$ hygromycin.

To generate transgenic $N$. benthamiana, the indicated plasmid was transformed into Agrobacterium strain GV3101. Agrobacterium-mediated N. benthamiana transformation was performed as previously described [43]. Transgenic plants were screened on 1/2 MS medium containing $50 \mathrm{mg} / \mathrm{l} \mathrm{Kanamycin.}$

2.3. Phytophthora capsici Culture Conditions, Culture Filtrate Acquisition and Inoculation Assay. The P. capsici strain LT263 used in this study was cultured and maintained at $25^{\circ} \mathrm{C}$ in $10 \%(v / v) \mathrm{V} 8$ juice medium (containing $0.1 \mathrm{M}$ $\mathrm{CaCO}_{3}$ ) in the dark. To produce $\mathrm{CF}$, mycelium was cultured in liquid $\mathrm{V} 8$ medium at $25^{\circ} \mathrm{C}$ for 3 days. The culture was then passed through a $22 \mu \mathrm{m}$ sterile filter unit (Merck Millipore, https://www.merckmillipore.com) to generate CF. For plant infiltration, a 1/10 CF solution was used. 
For mycelium inoculation, $N$. benthamiana leaves were inoculated by $5 \mathrm{~mm}$ disks of 4 -day growth mycelium at 24 hours postinfiltration. Lesion areas were measured and photographed under UV light at $48 \mathrm{hpi}$. For zoospore inoculation on leaves of Arabidopsis, P. capsici mycelium was incubated in liquid medium for 3 days and then washed 3 times with distilled water. Washed mycelium was incubated in sterilized water at $25^{\circ} \mathrm{C}$ in darkness for 12 hours. The cultures were cold shocked at $4^{\circ} \mathrm{C}$ for $20 \mathrm{~min}$ and incubated at $25^{\circ} \mathrm{C}$ for 2 hours to release zoospore. Leaves were soaked in 100 spores/ $\mu l P$. capsici zoospores for 30 minutes and then held under moist conditions for subsequent analysis by confocal microscopy.

Stock solution for trypan blue staining was produced by mixing trypan blue $(0.02 \mathrm{~g})$, glycerol $(10 \mathrm{ml})$, phenol $(10 \mathrm{~g})$, lactic acid $(10 \mathrm{ml})$, and sterilized water $(10 \mathrm{ml}) . \mathrm{N}$. benthamiana leaves were soaked in trypan stock solution for 24 hours at $24^{\circ} \mathrm{C}$. Leaves were then destained using ethanol for 5 days. Samples were put in ethanol for taking pictures under white light.

2.4. Bacterial Inoculation Assay. For bacterial inoculation, 5week-old N. benthamiana or 4-week-old Arabidopsis leaves were inoculated with Pst DC3000 or different DC3000 strains (AvrRPT2 or hopq $\left.q^{1-1}\right)\left(10^{6} \mathrm{cfu} / \mathrm{ml}\right)$. Bacterial population were calculated at $3 \mathrm{dpi}$.

2.5. Transient Expression in N. benthamiana. Transient expression in $N$. benthamiana was conducted as previous reported [44]. Briefly, Agrobacterium strains with mentioned constructs were cultured for 48 hours, collected, washed, and then resuspended in $10 \mathrm{mM} \mathrm{MgCl}_{2}$ to an optical density (OD) at $600 \mathrm{~nm}(0.4)$ and infiltrated into five-week-old $N$. benthamiana leaves.

2.6. $q R T-P C R$ Analysis. To perform real-time PCR, total RNA was extracted by a Total RNA Kit (Tiangen Biotech Co., Ltd., Beijing, China). The cDNAs were synthesized using the HiScript II Q RT SuperMix for qPCR (Vazyme Biotech Co., Ltd., Nanjing, China). Real-time PCR was performed by using an AceQ qPCR SYBR Green Master Mix (Vazyme Biotech Co., Ltd., Nanjing, China) on an ABI Prism Q5 system. AtUBQ10 or NbELF18 were used as internal references. Primers used for in real-time PCR are listed in Table S1. The qRT-PCR results were concluded from three biological replicates.

2.7. Western Blotting and Confocal Microscopy. Plant leaves for protein extraction were ground in liquid nitrogen. Extraction buffer $(0.1 \%$ Triton X-100, $150 \mathrm{mM}$ KCL, $50 \mathrm{mM}$ HEPES and $1 \mathrm{mM}$ EDTA, protease inhibitor cocktail; pH 7.5) and $1 \mathrm{mM}$ DTT was used for protein extraction. For Western blot assays, Flag, GFP (Abmart), and LUC (Sigma) antibodies were used.

To detect GFP accumulation after different treatments, confocal images were obtained at 12 hours after treatment by a confocal microscope (Zeiss LSM980, Germany). The average GFP florescence densities were quantified per 100 pixels of 20 randomly selected cells (relative unit) using ImageJ (https://imagej.en.softonic.com/).

\section{Results}

3.1. A Fluorescence- and Luminescence-Based Reporter System for the Function Investigation of $u O R F$ s. To investigate the function of uORFs, we designed a fluorescenceand luminescence-based reporter system to visibly measure the regulatory effect of uORFs (Figure 1(a)). In this system, the expression of green fluorescent protein (GFP) is under the control of mannopine synthase (MAS) promoter and the $5^{\prime}$ UTR of indicated gene. The luciferase (LUC) gene driven by CaMV $35 S$ promoter is used as an internal reference. GFP fluorescence and LUC luminescence intensities were quantified using a microplate reader. The relative fluorescence ratio (GFP/LUC) was calculated to remove perturbation resulted from agro-infiltration (Figure 1(a)). Notably, no significant interference was detected between green fluorescence and luminescence (Figure S1).

To check the reliability of our system, we used the previously reported cis translational repressor $5^{\prime}-\mathrm{UTR}_{\mathrm{TBF} 1}$ as a positive control $[31,34] .5^{\prime}-\mathrm{UTR}_{\mathrm{TBF} 1}$ significantly repressed GFP protein accumulation in our system (Figures 1(b)$1(d))$, indicating the effectiveness of this reporter system.

3.2. uORFs $_{A C D 11}$ Are Cis-Acting Elements That Repress Downstream Translation. Three uORFs were identified in the $5^{\prime}$-UTR ${ }_{\mathrm{ACD} 11}$ of Arabidopsis Col-0 ecotype and were named as uORF1, uORF2, and uORF3 (Figure 2(a), Figure S2a). The Col-0 uORF1 and uORF3 were found to be conserved in $96.6 \%$ and $100 \%$ of the 1,135 accessions in the uORFlight database, respectively (Figure S3a) [30]. Two major types of uORF2 could be identified in 1,135 accessions. Except Col- 0 type, Ws- 2 type uORF2 contained a synonymous nucleotide substitution ( $G$ to $C$ in the 60th base) (Figure S3a, S3b). All three $\mathrm{uORFs}_{\mathrm{ACD} 11}$ are highly conserved across Arabidopsis accessions which suggests that they may be functional.

A null mutant of $\mathrm{uORFs}_{\mathrm{ACD} 11}$ was created by mutating the start codon of all three ACD11 uORFs to CTG (hereafter "uorf1/2/3") and constructed into our vector. $5^{\prime}-\mathrm{UTR}_{\mathrm{ACD} 11}$ with native uORFs ${ }_{\mathrm{ACD} 11}$ (hereafter "NAT") was used as a control (Figure 2(b)). When expressed in $N$. benthamiana leaves, uorf1/2/3 exhibited much stronger GFP fluorescence than NAT. In contrast, they generated similar intensities of luminescence (Figure 2(b)). Similarly, GFP/LUC values showed that leaves expressing the uorf1/2/3 construct displayed significantly higher GFP fluorescence than those expressing NAT (Figure 2(c)). qRT-PCR and Western blot assays demonstrated that uorf $1 / 2 / 3$ and NAT constructs generate similar levels of GFP mRNA in $N$. benthamiana leaves (Figure 2(d)), but uorf1/2/3 expression leads to higher GFP protein accumulation (Figure 2(e)). These results indicate that $\mathrm{uORFs}_{\mathrm{ACD} 11}$ negatively regulate downstream protein accumulation at the translation level. Notably, the absence of $5^{\prime} \mathrm{UTR}_{\mathrm{ACD} 11}$ does not change GFP transcript, protein or fluorescence level as compared to uorf $1 / 2 / 3$ (Figures 2(b)-2(e)), indicating that the non-uORF regions in $5^{\prime}-\mathrm{UTR}_{\mathrm{ACD} 11}$ may not affect the expression of downstream protein. 


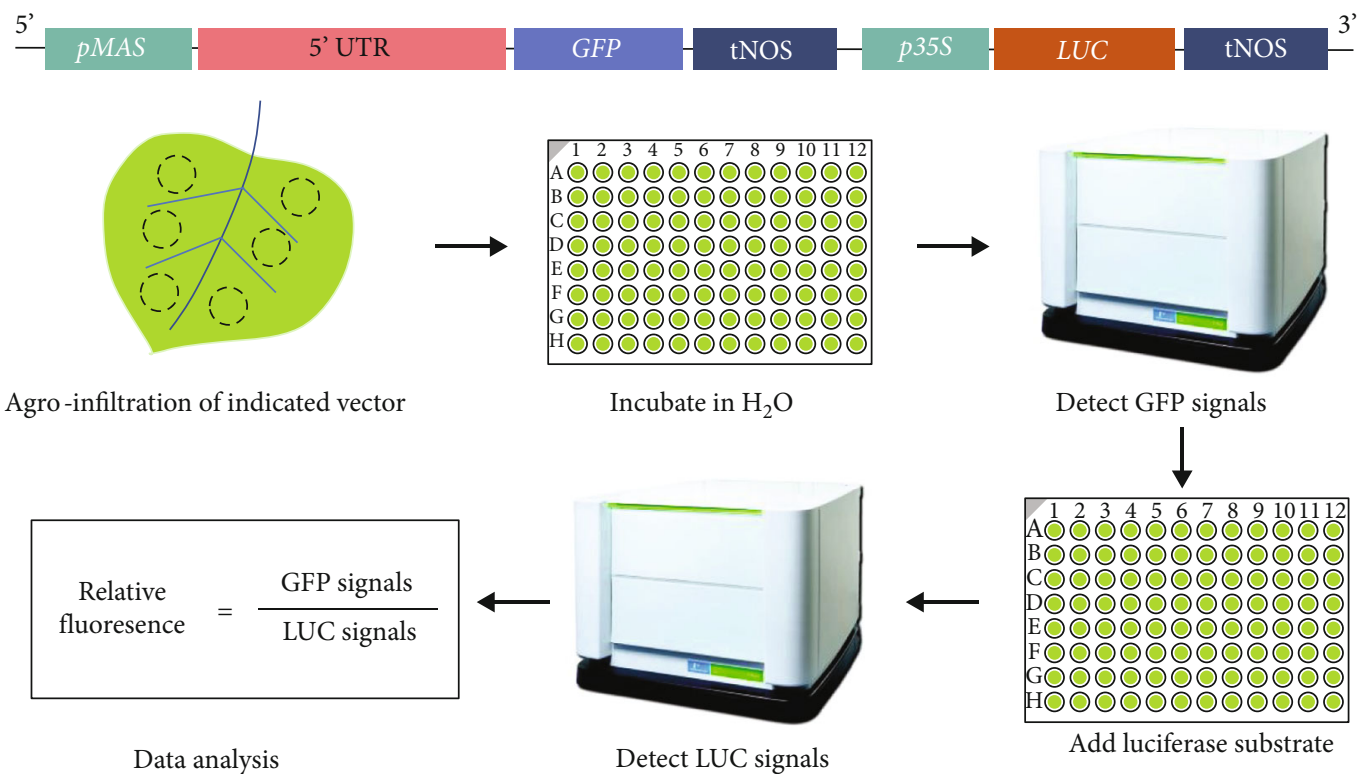

(a)

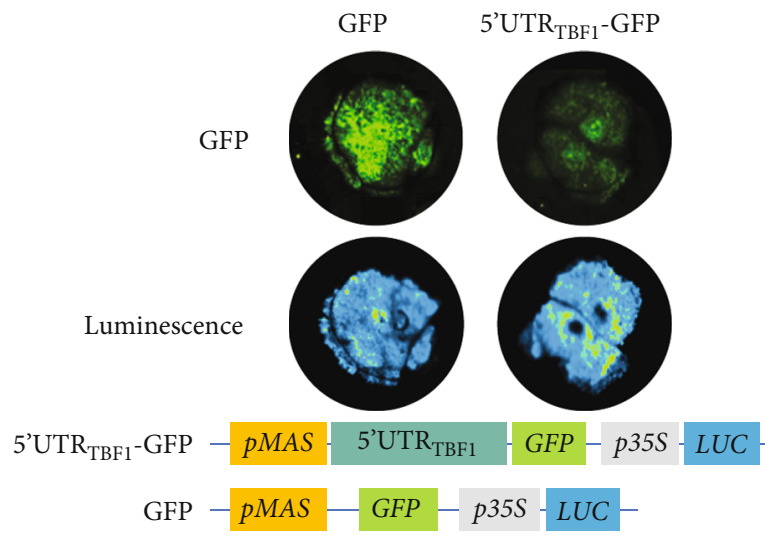

(b)

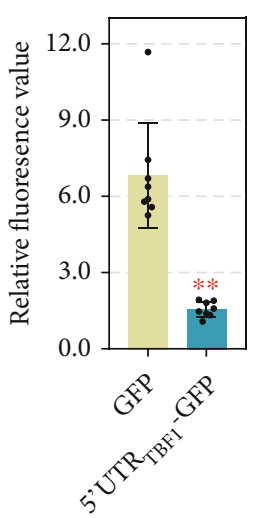

(c)

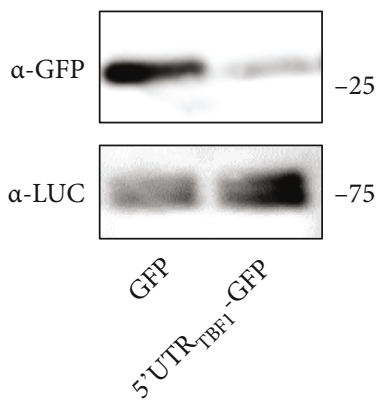

(d)

FIGURE 1: Establishment of a fluorescence- and luminescence-based reporter system to study the functions of uORFs. (a) Diagram of the fluorescence- and luminescence-based reporter system. Illustration of the constructed plasmids used in this study is shown at the top. Workflow of our system is shown below. (b) Repression of GFP production by the $5^{\prime}$-UTR of TBF1. Photos were taken 48 hours post agro-infiltration. Illustration of the indicated plasmids was shown below. (c) Relative fluorescence of leaves expressing indicated plasmids. Relative fluorescence was calculated based on the formula shown in (c) (mean $\pm \mathrm{SD} ; n=8$, Student's $t$-test, $P<0.01$ ). (d) Decreased GFP protein level confirmed by Western blot assay. The GFP protein accumulation was detected by $\alpha$-GFP antibody. Equal loading of each sample is indicated using the luciferase protein.

To test whether uORFs function as trans- or cis-elements, uorf $1 / 2 / 3$ construct was coinfiltrated with the constructs harboring coding cassettes of uORF1, uORF2, uORF3, or all of them. The NAT construct was used as control. GFP translation cannot be blocked via coexpression peptides of any individuals or all three uORFs (Figure 2(f )), demonstrating that uORFs act in cis.

\section{3. uORFs ${ }_{A C D 11}$ Have Variable Contributions to} Translational Repression. We next investigated the individual contributions of $\mathrm{uORFs}_{\mathrm{ACD} 11}$ to translational repression. A series of $\mathrm{uORFs}_{\mathrm{ACD} 11}$ mutants were created by individually or simultaneously mutating the start codon of uORF1,
uORF2, and/or uORF3 to CTG (Figure 3(a)). These mutants exhibited comparable luminescence signals but highly variable GFP fluorescence (Figure 3(a)). Despite their relatively weak Kozak strength (Figure S2b), all three $\mathrm{uORFs}_{\mathrm{ACD} 11}$ are involved in the translational repression of downstream gene (Figures 3(a)-3(d)). The presence of any single $\mathrm{uORF}_{\mathrm{ACD} 11}$ was able to sustain the inhibition phenotype (Figures 3(a)-3(d)). Among them, uORF1 tends to be the dominant inhibitory element. Disruption of uORF1 alone could significantly increase GFP signal, which was not observed on uORF2 or uORF3 disruption (Figures 3(a)$3(d)$ ). Nevertheless, a mutant with intact uORF3 and the disrupted uORF1 and uORF2 leads to the lowest GFP 


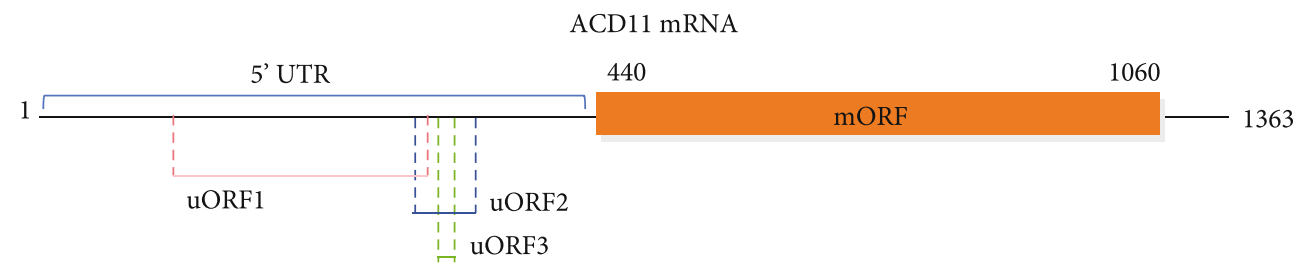

(a)
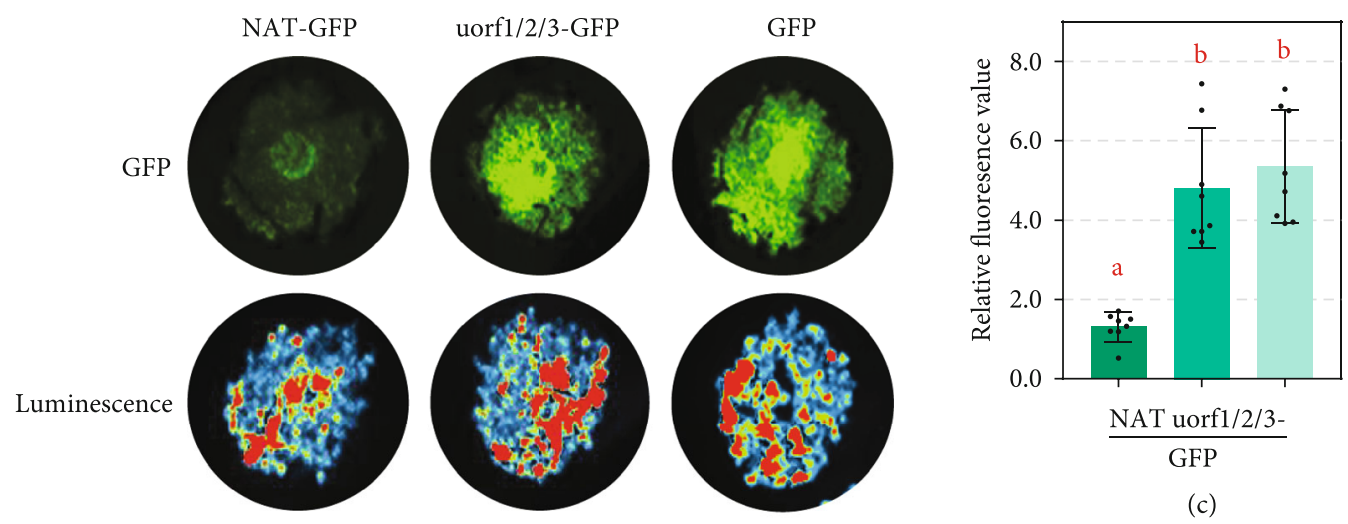

(c)
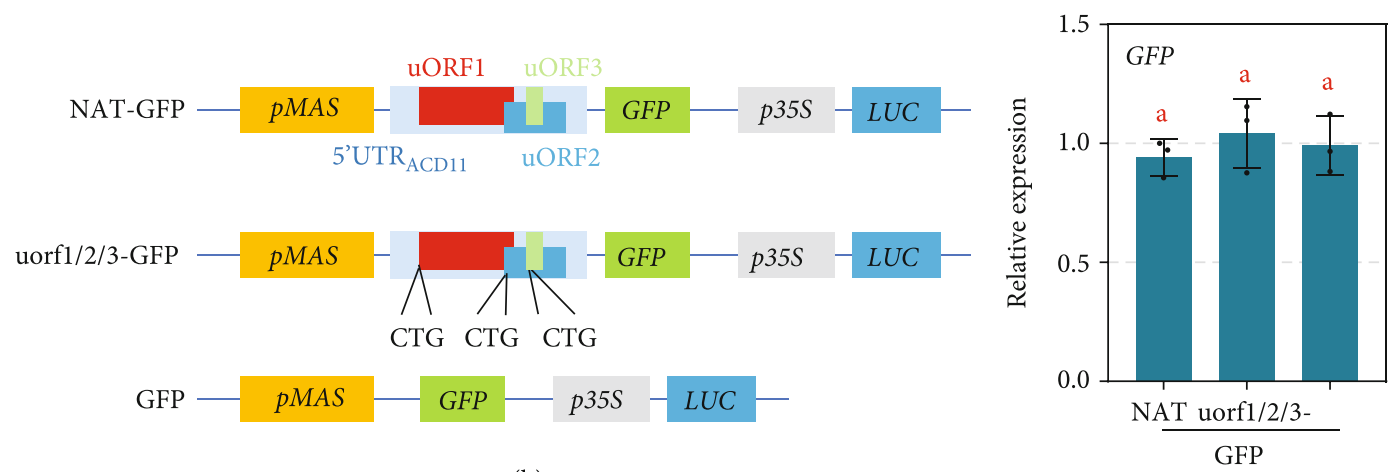

(b)

(d)

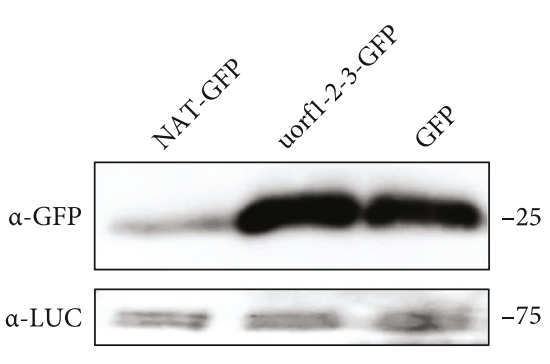

(e)

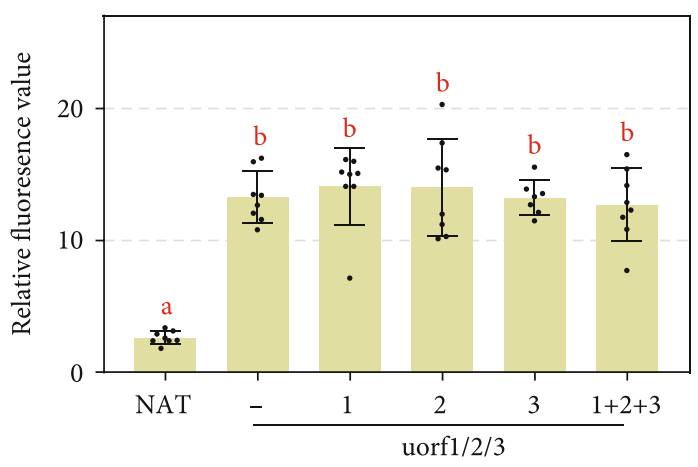

(f)

FIGURE 2: uORFs of $A C D 11$ are cis-acting elements that repress downstream translation. (a) Diagram of three uORFs identified in the $5^{\prime}$ UTR of ACD11. The main ORF (mORF) indicates ACD11. (b) Repression of GFP production by the $5^{\prime}$-UTR of $A C D 11$. Photos were taken 48 hours post agro-infiltration. Illustration of the indicated plasmids was shown below. (c) Relative fluorescence of leaves expressing indicated plasmids. Relative fluorescence was calculated based on the formula shown in Figure 1(c) (mean \pm SD; $n=8$, Student's $t$-test, $P$ <0.01). (d) Relative transcript accumulation levels of GFP. Transcript accumulation levels of GFP were analyzed by qRT-PCR with NbELF18 as internal reference. Bars represent standard errors from three biological replicates (mean \pm SD; $n=3 ;{ }^{* *}, P<0.01 ;$ Student's $t$ -test). (e) Decreased GFP protein expression confirmed by Western blot assay. The GFP protein accumulation was detected by $\alpha$-GFP antibody. Equal loading of each sample is indicated by the luciferase protein. (f) ACD11 uORFs act in cis. uORFs was expressed in trans in leaves expressing uorf1/2/3 plasmid. Relative fluorescence was calculated based on the formula shown in Figure $1(\mathrm{c})$ (mean \pm SD; $n \geq 8$ , Student's $t$-test, $P<0.01)$. 

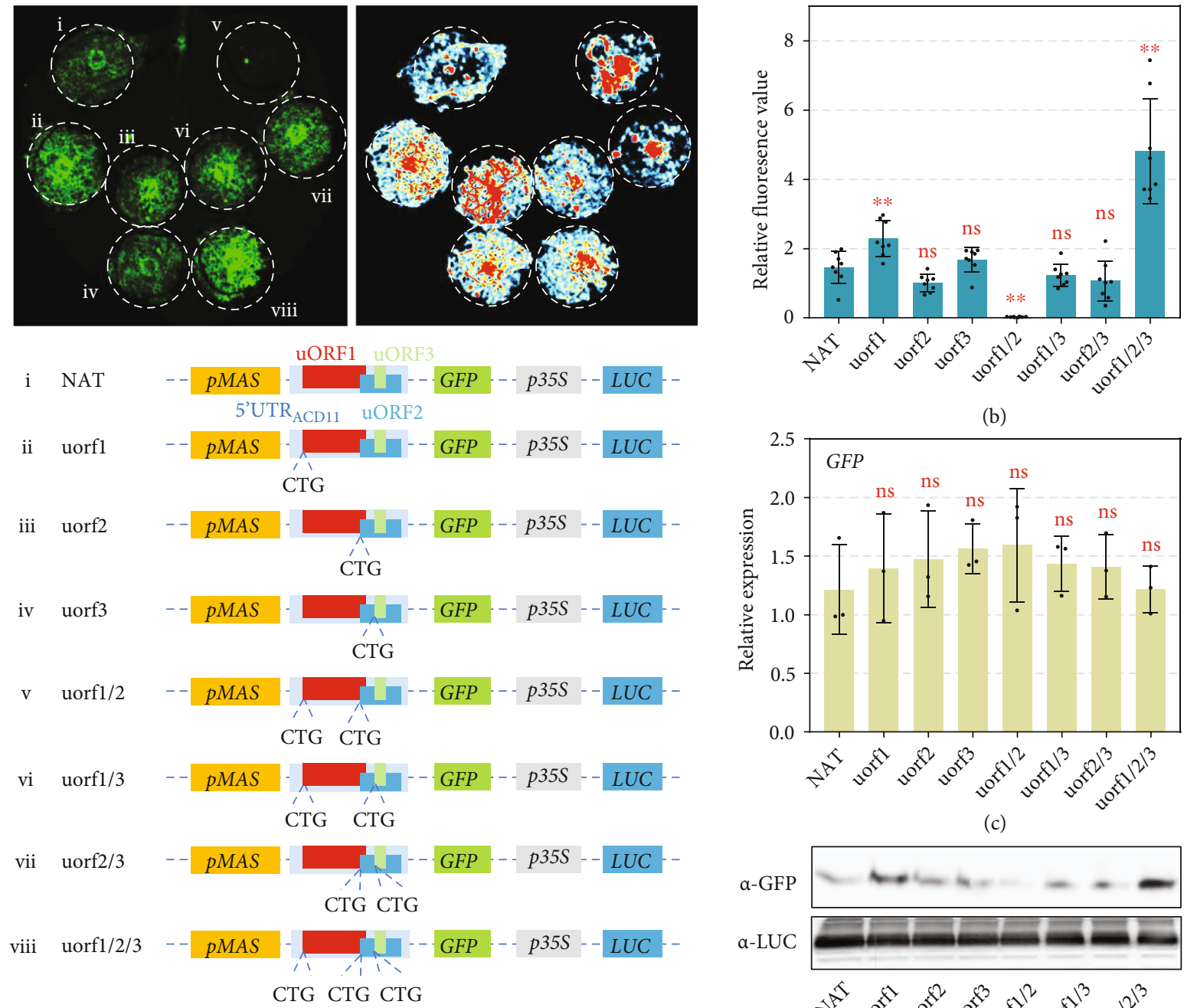

(b)

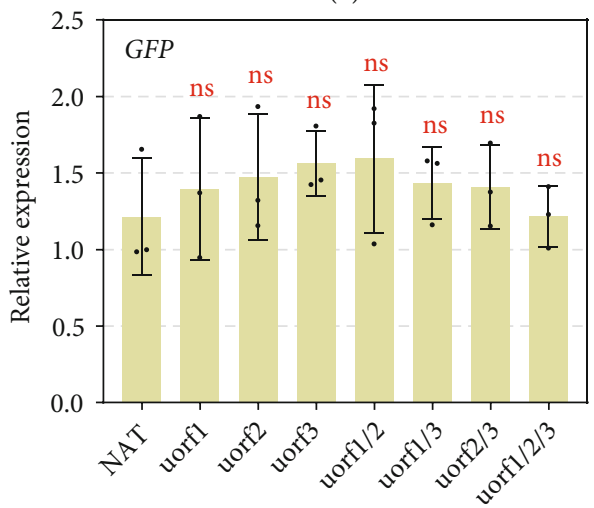

(c)

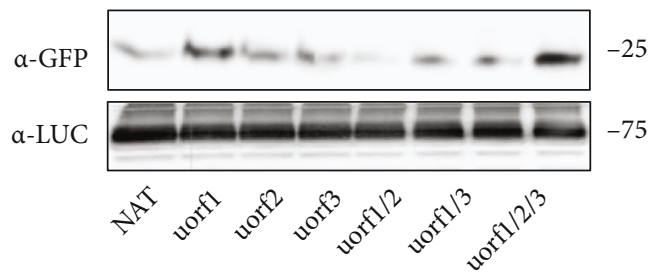

(a)

(d)

FIGURE 3: Various uORFs combinations show different downstream repressing efficiencies. (a) Repression of GFP production by ACD11 uORF mutants. Photos were taken 48 hours post agro-infiltration. Illustration of the indicated plasmids was shown below. (b) Relative fluorescence in leaves expressing indicated plasmids. Relative fluorescence was calculated based on the formula shown in Figure 1(c) (mean \pm SD; $n=8$, Student's $t$-test, $P<0.01$ ). (c) Relative transcript accumulation levels of GFP. Transcript accumulation levels of GFP were analyzed by qRT-PCR with $N b E L F 18$ as internal reference. Bars represent standard errors from three biological replicates (mean $\pm \mathrm{SD} ; n=3$; $^{* *}, P<0.01$; Student's $t$-test). (d) Decreased GFP protein expression confirmed by Western blot assay. The GFP protein accumulation was detected by $\alpha$-GFP antibody. Equal loading of each sample is indicated by the luciferase protein.

accumulation (Figures 3(a)-3(d)), indicating the shortest 9base-pair uORF3 has the strongest repression alone. The result is consistent with the report that uORF length is not correlated with the suppression capacity [45]. Taken together, these observations indicate that $\mathrm{uORFs}_{\mathrm{ACD} 11}$ have complex genetic interactions, and they are not simply additive in repressing downstream protein translation.

3.4. $\mathrm{UORFs}_{A C D 11}$-Mediated Translational Repression Is Attenuated upon Pathogen Infection. The $5^{\prime}-\mathrm{UTR}_{\mathrm{ACD} 11}-\mathrm{GFP}$ transgenic Arabidopsis plants were produced to test the response of $\mathrm{uORFs}_{\mathrm{ACD} 11}$ to pathogen infection (Figure S4a). GFP fluorescence generated in these transgenic plants was too weak to detect using in vivo fluorescence imaging
(Figure S4b), but visible under confocal microscope (Figure 4(a)). Transgenic Arabidopsis leaves were challenged with the oomycete pathogen Phytophthora capsici (Figure S4c). GFP fluorescence was enhanced in leaves inoculated with $P$. capsici zoospores or culture filter (CF) (Figures 4(a) and 4(b)) [46]. qRT-PCR analysis showed that the transcript accumulation level of GFP was unchanged after inoculation (Figure 4(c)). Western blot assay confirmed that the inoculation of either $P$. capsici zoospores or CF promoted GFP accumulation at the protein level (Figure 4(d)). A timecourse assay further showing that GFP protein accumulation increased over time in $5^{\prime}-\mathrm{UTR}_{\mathrm{ACD} 11}$-GFP transgenic Arabidopsis leaves infected by $P$. capsici zoospores (Figure 4(e)), indicating that the translational repression 


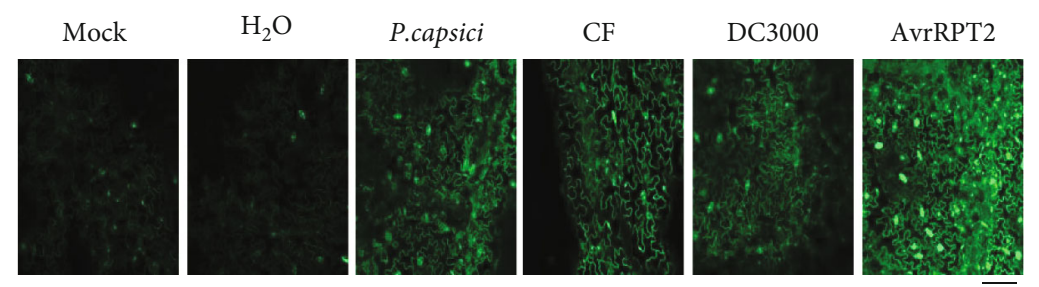

(a)

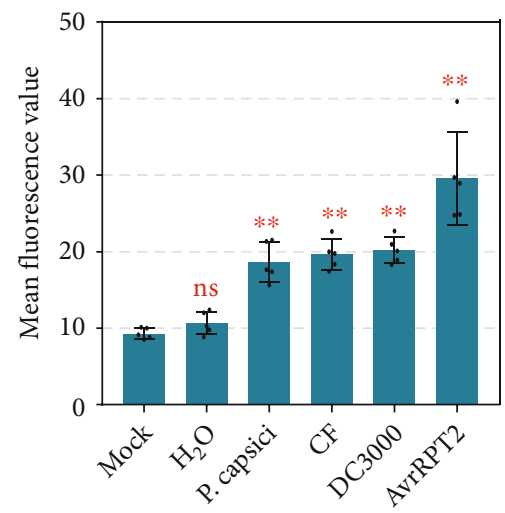

(b)

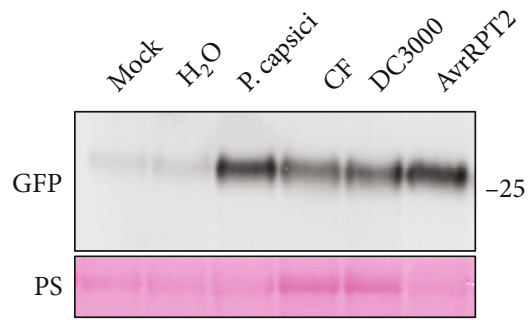

(d)

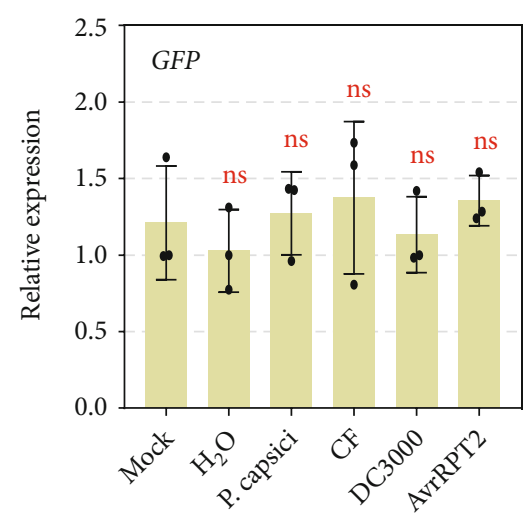

(c)

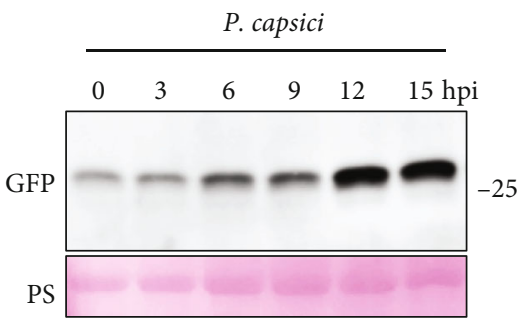

(e)

FIGURE 4: $\mathrm{uORF}_{\mathrm{ACD} 11}$-mediated translational repression is attenuated upon pathogen infection. (a) GFP accumulation after pathogenrelated treatments. Confocal microscope images were taken 12 hours posttreatment. Bar $=50 \mu \mathrm{m}$. (b) Mean fluorescence value of indicated leaves. The average GFP florescence densities were quantified per 100 pixels of 20 randomly selected cells (relative unit) using ImageJ. (c) Relative transcript accumulation levels of GFP. Transcript accumulation levels of GFP were analyzed by qRT-PCR with AtUBQ10 as internal reference. Bars represent standard errors from three biological replicates (mean $\pm \mathrm{SD} ; n=3$; ${ }^{* *}, P<0.01$; Student's $t$ -test). (d) GFP protein accumulation detected by Western blot assay. The $\alpha$-GFP antibody was used to detect the expression of GFP protein. Equal loading of each sample is indicated by Ponceau staining. (e) GFP protein accumulation changes after $P$. capsici inoculation. The GFP protein accumulation was detected by $\alpha$-GFP antibody. Equal loading of each sample is indicated by Ponceau staining.

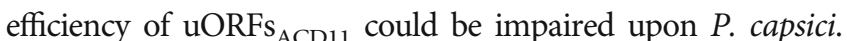
Similar to $P$. capsici, the bacterial pathogen Pseudomonas syringae pv. tomato (Pst) DC3000 also induced GFP accumulation in $5^{\prime}-\mathrm{UTR}_{\mathrm{ACD} 11}$-GFP transgenic Arabidopsis leaves at the translation level (Figures 4(a)-4(d)). Interestingly, Pst DC $3000_{\text {AvrRPT2 }}$, an avirulent strain that induces hypersensitive response in Arabidopsis [47, 48], showed higher GFP-induction efficiency than the wild-type (WT) Pst DC3000 (Figures 4(a)-4(d)). This observation indicates that the translational repression ability of $\mathrm{uORFs}_{\mathrm{ACD} 11}$ can be attenuated after plant recognizing different pathogens.

\subsection{Different $\mathrm{UORFs}_{\text {ACD11 }}$ Cassettes Lead to Variable} Accumulation of AtLecRK-VI.2 for Acquired Resistance. Since different $\mathrm{uORFs}_{\mathrm{ACD} 11}$ combinations showed variable downstream translational repression efficiencies, they could be used to fine-tune exogenous protein accumulation in transgenic plants, which may minimize the deleterious pleio- tropic effects caused by gene overexpression [13-15] and help to balance plant growth and immunity [34].

AtLecRK-VI.2, a positive modulator of PAMP-triggered immunity (PTI) response and SAR [40-42], was selected to test its translational regulation by $\mathrm{uORFs}_{\mathrm{ACD} 11}$ cassettes. Constructs of AtLecRK-VI.2 under the control of uorf2, uorf3, uorf1/2/3, or NAT were transiently expressed in $N$. benthamiana. Similar to results obtained using the GFP reporter, uorf1/2 led to the lowest AtLecRK-VI.2 protein accumulation. uorf2, uorf3, and NAT resulted in moderate protein accumulation levels while the highest AtLecRKVI.2 level was achieved by using uorf1/2/3 (Figure 5(a)). Consistent with the translational repression function of $\mathrm{uORFs}_{\mathrm{ACD} 11}$, no significant difference was observed on AtLecRK-VI.2 transcription levels (Figure 5(b)).

$P$. capsici resistance levels were tested for N. benthamiana plants transiently expressing AtLecRK-VI.2 under the control of different $\mathrm{uORFs}_{\mathrm{ACD} 11}$ cassettes. Compared to 


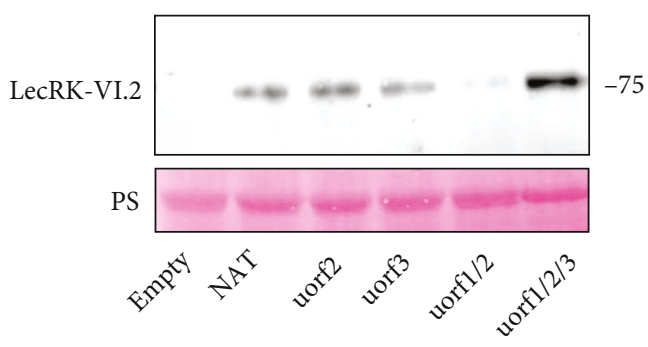

(a)

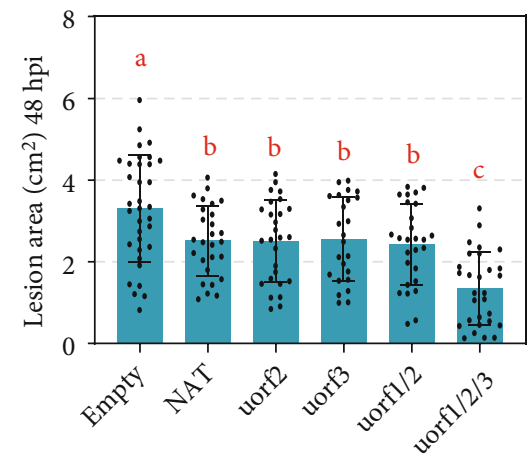

(c)

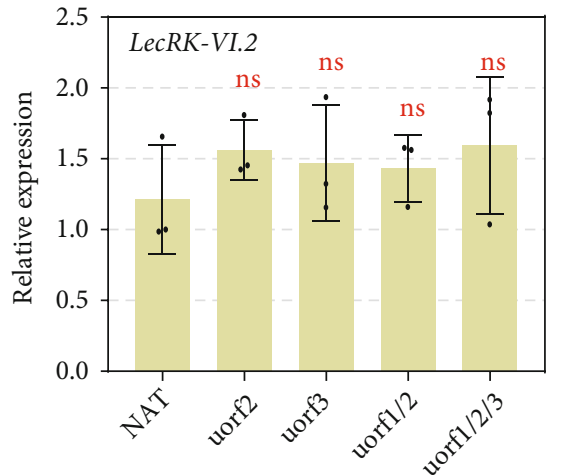

(b)

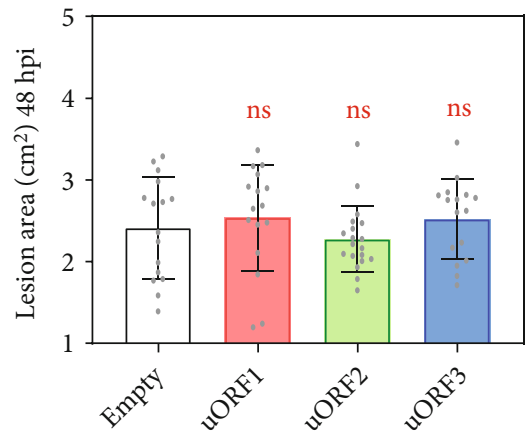

(d)

FIGURE 5: Different uORFs ${ }_{\text {ACD11 }}$ cassettes lead to variable AtLecRK-VI.2 protein accumulation and pathogen resistance levels. (a) AtLecRKVI.2 protein accumulation levels detected by Western blot assay. The $\alpha$-FLAG antibody was used to detect the expression of AtLecRK-VI.2 protein. Equal loading of each sample is indicated by Ponceau staining. (b) Relative transcript accumulation levels of AtLecRK-VI.2. Transcript accumulation levels of AtLecRK-VI.2 were analyzed by qRT-PCR with NbELF18 as internal reference. Bars represent standard errors from three independent biological replicates (mean $\pm \mathrm{SD} ; n=3$; ${ }^{*}, P<0.01$; Student's $t$-test). (c) Expression of AtLecRK-VI.2 driven by ACD11 uORF-mutants provides variable levels of resistance to $P$. capsici infection in $N$. benthamiana. Lesion areas were calculated at $48 \mathrm{hpi}$. The result was calculated from three independent replicates with at least five leaves per replicate (mean \pm SD; $n \geq 18$; **, $P<0.01$, Student's $t$-test). (d) No pathogen resistance is induced by uORF1, uORF2, or uORF3. Leaves were stained by trypan blue and destained with ethanol. Photos were taken in white light and shown in (a). Bar $=2 \mathrm{~cm}$. The lesion areas were calculated from three independent experiments were shown below (mean $\pm \mathrm{SD} ; n \geq 18$; ${ }^{*}, P<0.01$, Student's $t$-test).

empty vector, all cassettes significantly enhanced plant immunity to $P$. capsici at 48 hours postinoculation (hpi), with uorf1/2/3-LecRK-VI.2 delivering the highest resistance (Figure 5(c)). No increased resistance could be found in leaves expressing uORF1, uORF2, or uORF3 (Figure 5(d)), indicating that none of the peptides encoded by the three uORFs are directly involved in $P$. capsici resistance.

All expression constructs except uorf1/2-LecRK-VI.2 induced intense cell death at 5 days after infiltration (dpi) (Figure 6(a)), which may be explained by the lowest LecRK-VI.2 accumulation level caused by uorf $1 / 2$.

3.6. Combinations of Different $u O R F$ s and the NbPR1 Promoter Lead AtLecRK-VI.2 to Be Pathogen-Inducible. To express LecRK-VI.2 specifically in response to infection, we combined the pathogen-inducible $N$. benthamiana PR1 (NbPR1) promoter (pPR1) with different uORFs-LecRKVI.2 cassettes and transiently expressed them in $N$. benthamiana. $P$. capsici CF infiltration significantly enhanced LecRK-VI.2 transcript (Figure 6(b)) and protein (Figure 6(c)) accumulations in leaves expressing pPR1NAT-LecRK-VI.2, pPR1-uorf1/2-LecRK-VI.2, or pPR1-
uorf1/2/3-LecRK-VI.2 construct. Despite that pPR1-uorf1/ 2-LecRK-VI.2 led to the lowest LecRK-VI.2 protein accumulation, it provided similar level of $P$. capsici resistance as that of pPR1-NAT-LecRK-VI.2 (Figure 6(d)). Intense cell death was induced at $5 \mathrm{dpi}$ in leaves expressing pPR1-uorf1/2/3LecRK-VI.2 but not pPR1-uorf1/2-LecRK-VI.2 (Figure 6(a)). However, unlike NAT-LecRK-VI.2, expression of pPR1-NAT-LecRK-VI.2 did not cause necrosis in leaves, indicating that the activity of pathogen-inducible $p P R 1$ may confer a lower downstream gene expression level in normal conditions (Figure 6(a)).

3.7. pPR1-uorf1/2-AtLecRK-VI.2 Enhances N. benthamiana Resistance to $P$. capsici with no Apparent Suppression to Plant Growth. Stable transgenic N. benthamiana lines expressing pPR1-NAT-LecRK-VI.2, pPR1-uorf1/2-LecRKVI.2, or pPR1-uorf1/2/3-LecRK-VI.2 were created for functional analysis of the pPR1-uORFs ${ }_{\mathrm{ACD} 11}$ cassettes (Figure S5). Consistent with a previous report that transgenic $N$. benthamiana plants expressing AtLecRKVI.2 exhibit normal growth phenotypes [42], no retarded growth was found in $N$. benthamiana lines expressing 


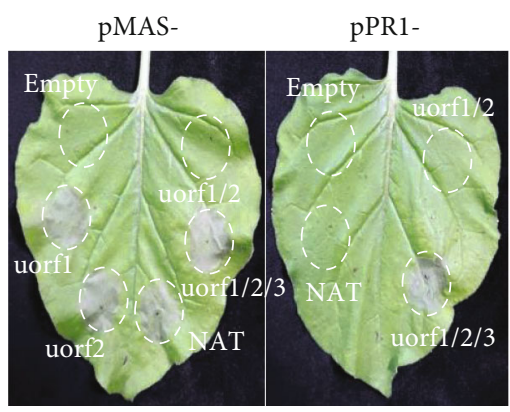

(a)

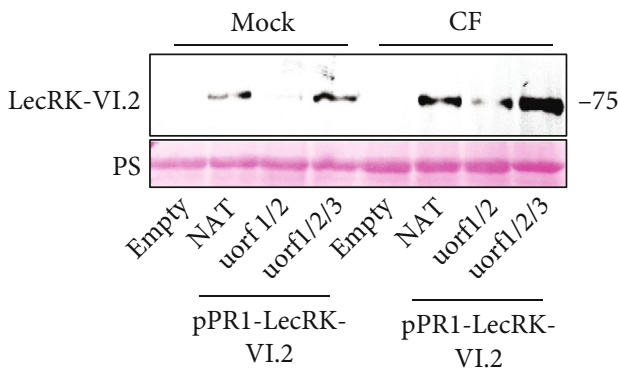

(c)

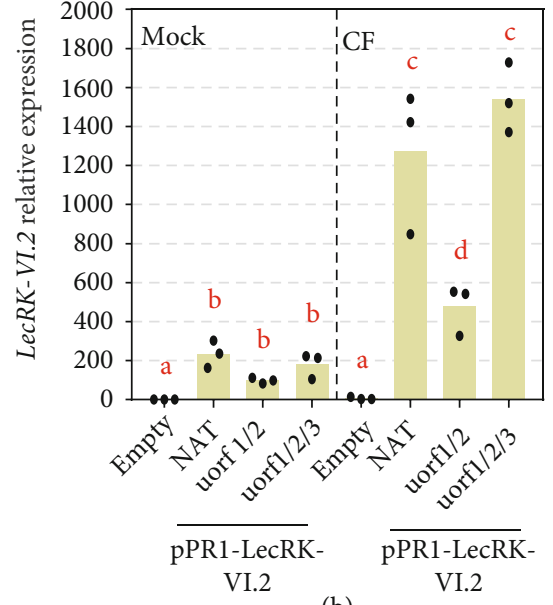

(b)

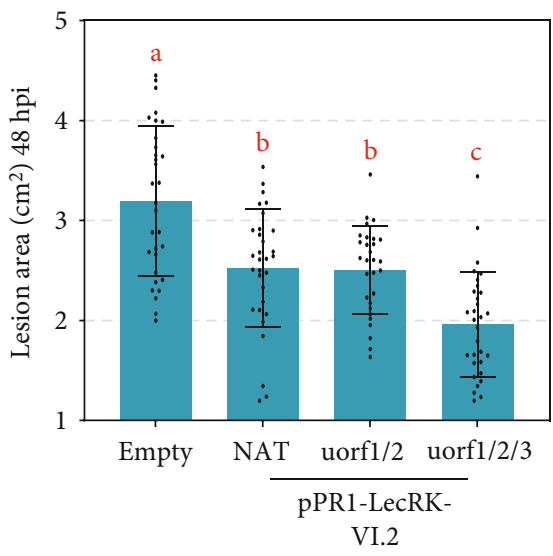

(d)

FIgURE 6: Pathogen-inducible protein accumulation of AtLecRK-VI.2 is achieved by using the combinations of different uORFs and the NbPR1 promoter. (a) Cell death induced by indicated gene products. Leaves infiltrated with Agrobacterium harboring indicated constructs. Photos were taken at $5 \mathrm{dpi}$ in white light. (b) CF-induced AtLecRK-VI.2 transcript accumulation confirmed by qRT-PCR. Transcript accumulation levels of AtLecRK-VI.2 were analyzed by qRT-PCR. P. capsici CF was infiltrated into leaves expressing the indicated plasmid. Total RNA was extracted 1 hour posttreatment. The NbELF18 gene was used as an internal reference. Bars represent standard errors from three biological replicates (mean $\pm \mathrm{SD} ; n=3$; ${ }^{*}, P<0.01$; Student's $t$-test). (c) Induced AtLecRK-VI.2 protein accumulation confirmed by Western blot assay. CF was infiltrated into leaves expressing the indicated plasmid. Proteins was extracted 2 hours posttreatment. The $\alpha$-FLAG antibody was used to detect the expression of AtLecRK-VI.2 protein. Equal loading of each sample is indicated by Ponceau staining. (d) Enhanced resistance to P. capsici infection provided by ACD11 uORF-mutants coupled with the PR1 promoter and AtLecRK-VI.2 in N. benthamiana. Lesion areas were calculated from three independent experiments with at least five leaves per replicate (mean $\pm \mathrm{SD} ; n \geq 18 ;{ }^{* *}, P<0.01$, Student's $t$-test).

pPR1-NAT-LecRK-VI.2 or pPR1-uorf1/2-LecRK-VI.2 (Figures $7(\mathrm{a})$ and $7(\mathrm{~b})$ ). Notably, stable expression of pPR1-uorf1/2/3-LecRK-VI.2 in $N$. benthamiana resulted in growth inhibition (Figures $7(\mathrm{a})$ and $7(\mathrm{~b})$ ). Consistent with the case in $A$. thaliana, treatment of leaves with $\mathrm{CF}$ resulted in a significant induction of LecRK-VI.2 transcription levels in all three stable transgenic lines (Figure $7(\mathrm{c})$ ). Upon inoculation of leaves using $P$. capsici mycelium (Figure $7(\mathrm{~d})$ ), LecRK-VI.2 protein accumulation also increased by variable levels in the three transgenic lines (Figure $7(\mathrm{e})$ ). uorf1/2, NAT, and uorf1/2/3 showed relatively strong, moderate, and weak translational repression efficiencies, respectively (Figure $7(\mathrm{e})$ ). These results indicate that the $\mathrm{PPR} 1-\mathrm{uORFs} \mathrm{ACD}_{\mathrm{Al}}$ cassettes can fine-tune downstream gene expression and translation in a pathogen-inducible manner.

In resistance assay, all three stable transgenic lines exhibited enhanced resistance to both $P$. capsici (Figure $7(\mathrm{f})$ ) and Pst DC3000 hopq1-1 (Figure 7(g)) as compared to wildtype $N$. benthamiana plants, with pPR1-uorf1/2/3-LecRK-VI.2 delivering strongest protection.

LecRK-VI.2 functions as an extracellular pyridine nucleotide receptor involved in SAR [41]. To test whether the observed alteration protein levels in the three transgenic line affected SAR response, we assessed the ability of DC3000 $h o p q^{1-1}$ to colonize upper leaves three days after the inoculation of lower leaves. Systemic resistance was observed in wildtype and the three transgenic lines (Figure $7(\mathrm{~h})$ ). 

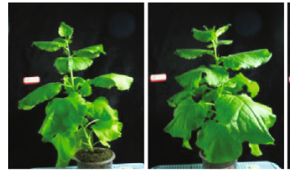

$\mathrm{Nb}$

NAT

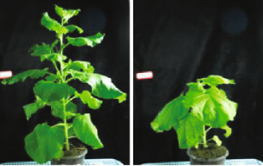

uorf1/2 uorf1/2/3

(a)
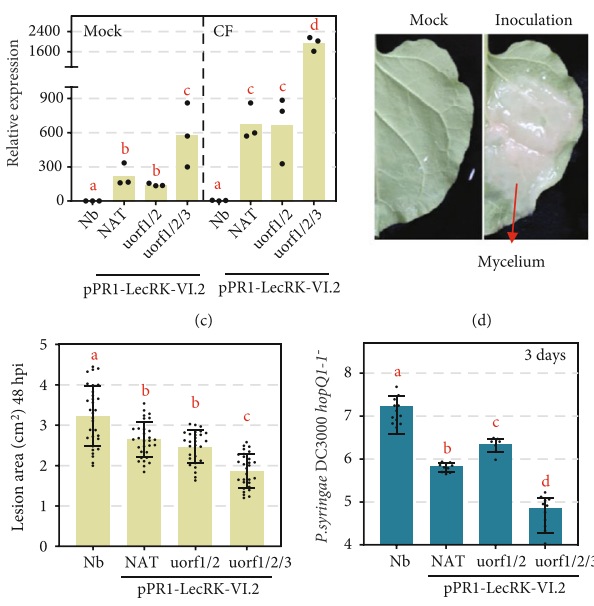

Mycelium

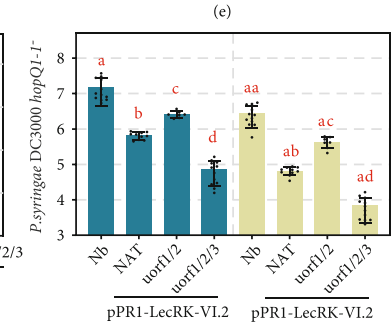

Local tissue

Systemic tissue

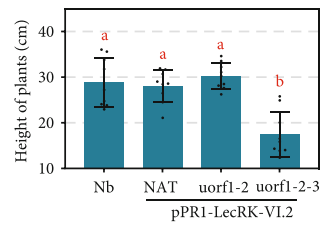

(b)

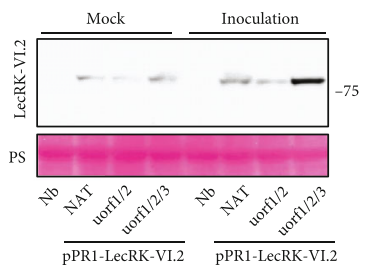

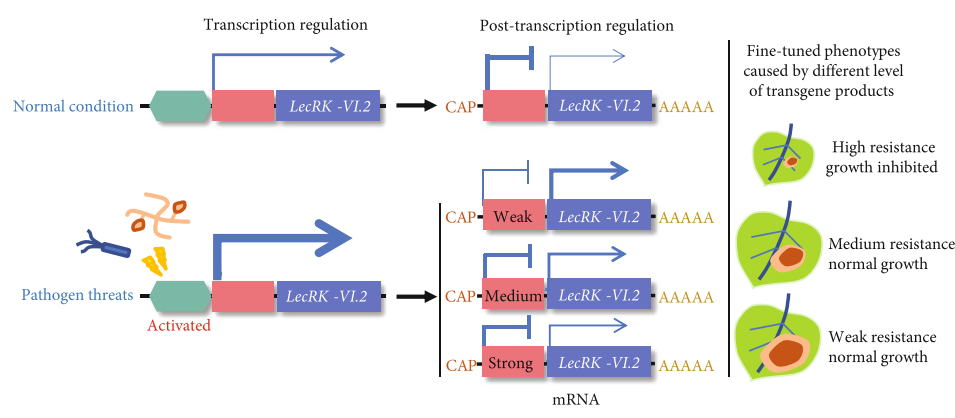

PR1 promoter $\mathrm{uORF}_{\mathrm{ACD} 11}$ cassette Weak Medium
Bacteria pathogens

CAP-: 5 ' CAP of mRNA

-AAAAA: Poly(A) of mRNA

(i)

FIGURE 7: pPR1-uORFs ${ }_{\mathrm{ACD} 11}$ cassettes fine-tune AtLecRK-VI.2-mediated resistance to pathogens in transgenic N. benthamiana. (a, b) The growth phenotypes of indicated transgenic $N$. benthamiana plants. Photos were taken at 8 weeks after sowing (a). Plant heights (from soil to the top of plants) were calculated and showed in (b). (c) AtLecRK-VI.2 transcript accumulation confirmed by qRT-PCR. Transcript accumulation levels of AtLecRK-VI.2 were analyzed by qRT-PCR. CF was infiltrated into the indicated leaves. Total RNA was extracted 1 hour posttreatment. The NbELF18 gene was used as an internal reference. Bars represent standard errors from three biological replicates (mean $\pm \mathrm{SD} ; n=3{ }^{* *}, P<0.01$; Student's $t$-test). (d, e) Induced AtLecRK-VI.2 protein accumulation upon $P$. capsici infection. $P$. capsici mycelium was inoculated on the indicated leaves. Proteins was extracted 12 hours postinoculation. The $\alpha$-FLAG antibody was used to detect the expression of AtLecRK-VI.2 protein. Equal loading of each sample is indicated by Ponceau staining. (f) Enhanced resistance to $P$. capsici infection in transgenic $N$. benthamiana. Lesion areas were calculated from three independent experiments with at least five leaves per replicate (mean $\pm \mathrm{SD} ; n \geq 18 ;{ }^{* *}, P<0.01$, Student's $t$-test). (g) Fine-tuning resistance to $P$. syringae hopq ${ }^{1-1}$ in transgenic plants. Error bars represent the mean $\pm \mathrm{SD} ; n=3$; lowercase letters indicate statistical significance tested between multiple groups by oneway ANOVA at $P<0.05$. (h) Fine-tuning systemic acquired resistance to $P$. syringae hop $q^{1-1}$ in transgenic plants. Three lower leaves on each 4-week-old soil-grown plant were infiltrated with $\mathrm{H}_{2} \mathrm{O}$ or $P$. syringae hopq ${ }^{1-1}$ suspension $(\mathrm{OD}=0.0001)$. Two systemic leaves were challenge-inoculated with a $P$. syringae hop $q^{1-1}$ suspension $(\mathrm{OD}=0.0001)$ at 24 hours after the last infiltration. Three days later, eight leaves were collected to examine pathogen growth. Error bars represent the mean $\pm \mathrm{SD} ; n=3$; lowercase letters indicate statistical significance tested between multiple groups by one-way ANOVA at $P<0.05$. (i) A model for the usage of uORFs $\mathrm{ACD}_{11}$ in precisely engineering crop disease resistance at required levels to minimize negative impacts on plant growth. $\mathrm{uORFs}_{\mathrm{ACD} 11}$ inhibits downstream gene translation in normal conditions and releases it upon pathogens. uORFs ${ }_{\mathrm{ACD} 11}$ coupled with PR1 promoter can confer fine-tuned resistance in transgenic plants specifically when plants are facing pathogen threats. 
However, none of the three transgenic lines exhibited a proportionally stronger SAR response from controls (Figure 7(h)).

\section{Discussion}

Fine-tuning of quantitative traits is highly valued by breeders as it affords a sound approach to harness useful characteristics for breeding without serious field impairment $[49,50]$. Naturally occurred weak alleles affecting important traits have contributed to great advances in domestication, evolution, and breeding [51,52], but their utilization is restricted by low availability. Ectopic expression of foreign genes is an alternative approach to introduce the desired traits. However, overexpression can lead to undesirable phenotypes. For example, ectopic expression of AtNPR1 in rice using the maize ubiquitin promoter resulted in abnormal plant development and a reduction in seed size under some conditions [17]. Constitutive expression of the coding region of tobacco $t b z 17$ (Nttbz17) results in plants with thicker leaves [53]. Here, we successfully controlled the transgene products by using $\mathrm{uORFs}_{\mathrm{ACD} 11}$ and $\mathrm{PR} 1$ promoter to achieve a fine-tuned resistance level without growth retardation in transgenic plants (Figure 7(i)).

A uORF is a small ORF containing a start codon located upstream of their regulated gene. The three uORFs in $5^{\prime}$ $\mathrm{UTR}_{\mathrm{ACD11}}$ are conserved among Arabidopsis accessions, indicating their important regulatory roles. Their variable translational repression efficiencies may at least partially depend on the Kozak sequence context around their start codons [54]. Despite their relatively low Kozak strength, the three $\mathrm{uORFs}_{\mathrm{ACD} 11}$ effectively repress the translation of downstream gene in a redundant manner. Among them, uORF3 has the shortest length of 9 base pairs but the most favorable Kozak strength, which leads to its strongest translational repression efficiency. This observation explains why the uorf $1 / 2$ cassette has strong repression capacity and is suitable for fine-tuning transgene expression and balancing plant growth and immunity.

Natural uORFs has been successfully used in engineering plant defense. Ectopic expression of AtNPR1 driven by 35S:uORFs AtTBF1 $_{\text {renders broad-spectrum disease resistance }}$ to rice plants with no apparent growth retardation [34]. The potential value of edited uORFs is also emerging gradually. For example, uORF engineering of an important enzyme in vitamin C synthesis, LsGGP2, improves the tolerance of lettuce to oxidation stress and ascorbate content [55]. Here, we have shown that a modified uORF, uORF$\mathrm{s}_{\mathrm{ACD11}}$, can be used to down regulate gene translation under normal growth conditions while enabling the activation of translation when a pathogen is detected.

$A C D 11$ encodes a phingosine transfer protein. Its knockout causes activation of defense response and programmed cell death (PCD), indicating that ACD11 negatively regulates plant immunity [35]. The protein stability of ACD11 is also regulated by the E3 ligase XBA35.2 and its binding partners of BPL family proteins $[36,37]$. The three uORFs characterized in this study may be an additional control layer of ACD11 protein accumulation. In plants, the protein levels of such negative regulators are strictly controlled by multiple layers of mechanisms. Other examples of this multilayered regulation include the pentatricopeptide repeats proteinlike (PPRL) protein negatively regulates RESISTANT TO P. SYRINGAE 2- (RPS2-) mediated resistance pathway and is downregulated by a natural antisense short interfering RNA (nat-siRNA) derived from Arabidopsis GTPBINDING 2 (ATGB2) gene (nat-siRNAATGB2) at the transcription level [56]. EDS1-interacting J protein 1 (EIJ1) plays a negative role in plant defense response and its protein accumulation is restricted during pathogen infection process [57].

Based on our data, $\mathrm{uORFs}_{\mathrm{ACD} 11}$-mediated translational repression was attenuated upon either PAMP treatment or pathogen inoculation. Similar phenomenon has been reported in multiple other genes such as $\mathrm{uORF}_{\mathrm{TBF} 1}[31,58$, 59]. The R-motif, a highly enriched consensus sequence consisting of mostly purines, can be found in the $5^{\prime}$-UTR of these genes. However, no R-motif can be identified from $5^{\prime}$ $\mathrm{UTR}_{\mathrm{ACD} 11}$, indicating that $A C D 11$ and R-motif containing genes like TBF1 may respond to pathogen infection via distinct mechanisms. Additionally, the translational repression ability of $\mathrm{uORFs}_{\mathrm{ACD} 11}$ become weaker after the avirulent strain treatment. Avirulent strains lead to effector-triggered immunity (ETI) in host cells. Compared with basal resistance, ETI induces a stronger and faster defense response against pathogens. We speculated that the inhibition of $\mathrm{uORFs}_{\mathrm{ACD} 11}$-mediated translational repression ability was a part of defense response, and thus, $\mathrm{uORFs}_{\mathrm{ACD} 11}$-mediated translational repression was weaker upon avirulent strains.

The integration of $p P R 1$ is an improvement to the $\mathrm{uORFs}_{\mathrm{ACD11}}$ cassettes. The adoption of pathogen-inducible promoters is a logical solution to reduce growth distortion triggered by defense-related transgenes $[19,20]$, but they should be carefully selected for genetic engineering to avoid those with unfavorable auto-activation effect $[24,25]$. In this study, no obvious auto-activation is detected for $p P R 1$ uORFs $_{\mathrm{ACD} 11}$ cassettes and the strategy successfully avoids potential growth retardation induced by AtLecRK-VI.2.

In this study, we fine-tune AtLecRK-VI.2 expression via the combinations of $\mathrm{pPR} 1$ and $\mathrm{uORFs} \mathrm{ACD}_{11}$ cassettes. In this way, AtLecRK-VI.2 expression is strictly induced by pathogen infection and optimized to provide satisfactory resistance protection with no apparent impair on plant growth. These cassettes can be used for fine-tuning other genes of interest. They would be a useful toolkit for precisely engineering crop disease resistance and other important agronomic traits.

\section{Data Availability}

The TAIR locus IDs for genes mentioned in this study are AT5G01540 (AtLecRK-VI.2), AT2G34690 (AtACD11), and AT4G36990 (AtTBF1).

\section{Additional Points}

Highlight. The uORFsACD11 cassettes are demonstrated to be a useful toolkit for engineering crop disease resistance 
to desired levels and minimizing negative impact towards plant growth.

\section{Conflicts of Interest}

The authors declare that there is no conflict of interest regarding the publication of this article.

\section{Authors' Contributions}

$\mathrm{DD}, \mathrm{AX}$, and GA conceived and designed the project, jointly performed data analysis, and wrote the manuscript. GA, JL, XF, TL, HZ, JL, and WP performed the experiments. DS and $\mathrm{MJ}$ analysed data. DD and YZ wrote and modified the manuscript. All authors read and approved the final manuscript. Gan Ai and Jin Liu contributed equally to this work.

\section{Acknowledgments}

We thank Dr. Meixiang Zhang at Nanjing Agricultural University and Dr. Xiangxiu Liang at Chinese Agricultural University for their constructive suggestions. The work was supported by the National Natural Science Foundation of China (31625023, 31721004, and 32072507) and the Fundamental Research Funds for the Central Universities (KYT202001).

\section{Supplementary Materials}

Figure S1: no interference is detected between green fluorescence and luminescence. Leaves were agro-infiltrated with GFP or LUCIFERASE for 48 hours. Photos were taken by a cooled charge-coupled imaging apparatus. Figure S2: the three uORFs identified in the $5^{\prime}$-UTR of ACD11. (a) Detail sequence information of the three uORFs. (b) Kozak strength of uORFsACD11 and mORFACD11. Different start codon types with related Kozak strength are shown in the left. Start codon sequences with related Kozak strength is shown in the right. Figure S3: variation of uORFsACD11 in 1,135 Arabidopsis accessions. (a) Percentages of different uORF types in 1,135 Arabidopsis accessions. (b) uORF2 sequences of Col- 0 and 10 popular accessions. Figure S4: validation of transgenic Arabidopsis. (a) Validation of transgenic Arabidopsis. The designation of primers is shown at the top. PCR results using the primers is shown below. (b) GFP signal of transgenic leaves detected by in vivo fluorescence imaging system. Bright field image is shown on the left. Image taken by GFP channel is shown on the right. (c) A schematic diagram illustrating the experiment designation. Figure S5: validation of transgenic plants. The designation of primers is shown at the top. PCR results using the primers are shown below. Table S1: list of primers. (Supplementary Materials)

\section{References}

[1] F. Hartung and J. Schiemann, "Precise plant breeding using new genome editing techniques: opportunities, safety and regulation in the EU," Plant Journal, vol. 78, no. 5, pp. 742-752, 2014.
[2] M. Kashtwari, S. Mansoor, A. A. Wani et al., "Random mutagenesis in vegetatively propagated crops: opportunities, challenges and genome editing prospects," Molecular Biology Reports, vol. 24, 2021.

[3] C. Rato, M. F. Carvalho, C. Azevedo, and P. R. Oblessuc, "Genome editing for resistance against plant pests and pathogens,” Transgenic Research, vol. 30, no. 4, pp. 427-459, 2021.

[4] O. X. Dong and P. C. Ronald, "Genetic engineering for disease resistance in plants: recent progress and future perspectives," Plant Physiology, vol. 180, pp. 26-38, 2019.

[5] E. C. Oerke and H. W. Dehne, "Safeguarding production losses in major crops and the role of crop protection," Crop Protection, vol. 23, pp. 275-285, 2004.

[6] J. B. Ristaino, P. K. Anderson, D. P. Bebber et al., "The persistent threat of emerging plant disease pandemics to global food security," Proceedings of the National Academy of Sciences of the United States of America, vol. 118, no. 23, p. e2022239118, 2021.

[7] P. Christou, "Plant genetic engineering and agricultural biotechnology 1983-2013," Trends in Biotechnology, vol. 31, pp. 125-127, 2013.

[8] H. J. Schouten, F. A. Krens, and E. Jacobsen, "Cisgenic plants are similar to traditionally bred plants - international regulations for genetically modified organisms should be altered to exempt cisgenesis," EMBO Reports, vol. 7, pp. 750-753, 2006.

[9] S. J. Gurr and P. J. Rushton, "Engineering plants with increased disease resistance: what are we going to express?," Trends in Biotechnology, vol. 23, pp. 275-282, 2005.

[10] K. Bouwmeester, M. Han, R. Blanco-Portales et al., "The Arabidopsis lectin receptor kinase LecRK-I.9 enhances resistance to Phytophthora infestans in Solanaceous plants," Plant Biotechnology Journal, vol. 12, pp. 10-16, 2014.

[11] S. Lacombe, A. Rougon-Cardoso, E. Sherwood et al., "Interfamily transfer of a plant pattern-recognition receptor confers broad- spectrum bacterial resistance," Nature Biotechnology, vol. 28, no. 4, pp. 365-369, 2010.

[12] B. Schwessinger, O. Bahar, N. Thomas et al., "Transgenic expression of the dicotyledonous pattern recognition receptor EFR in rice leads to ligand-dependent activation of defense responses," PLoS Pathogens, vol. 11, no. 3, p. e1004809, 2015.

[13] H. Schoof, M. Lenhard, A. Haecker, K. F. Mayer, G. Jurgens, and T. Laux, "The stem cell population of Arabidopsis shoot meristems in maintained by a regulatory loop between the CLAVATA and WUSCHEL genes," Cell, vol. 100, pp. 635$644,2000$.

[14] J. Vrebalov, D. Ruezinsky, V. Padmanabhan et al., "A MADSbox gene necessary for fruit ripening at the tomato ripeninginhibitor (Rin) locus," Science, vol. 296, pp. 343-346, 2002.

[15] C. Xu, K. L. Liberatore, C. A. MacAlister et al., "A cascade of arabinosyltransferases controls shoot meristem size in tomato," Nature Genetics, vol. 47, no. 7, pp. 784-792, 2015.

[16] M. S. Chern, H. A. Fitzgerald, R. C. Yadav, P. E. Canlas, X. N. Dong, and P. C. Ronald, "Evidence for a diseaseresistance pathway in rice similar to the NPR1-mediated signaling pathway in Arabidopsis," Plant Journal, vol. 27, pp. 101-113, 2001.

[17] H. A. Fitzgerald, M. S. Chern, R. Navarre, and P. C. Ronald, "Overexpression of (At)NPR1 in rice leads to a BTH- and environment-induced lesion-mimic/cell death phenotype," Molecular Plant-Microbe Interactions, vol. 17, pp. 140-151, 2004. 
[18] J. Quilis, G. Penas, J. Messeguer, C. Brugidou, and B. S. Segundo, "The Arabidopsis AtNPR1 inversely modulates defense responses against fungal, bacterial, or viral pathogens while conferring hypersensitivity to abiotic stresses in transgenic rice," Molecular Plant-Microbe Interactions, vol. 21, pp. 1215-1231, 2008.

[19] D. B. Collinge, H. J. Jorgensen, O. S. Lund, and M. F. Lyngkjaer, "Engineering pathogen resistance in crop plants: current trends and future prospects," Annual Review of Phytopathology, vol. 48, pp. 269-291, 2010.

[20] B. B. Wulff, D. M. Horvath, and E. R. Ward, "Improving immunity in crops: new tactics in an old game," Current Opinion in Plant Biology, vol. 14, pp. 468-476, 2011.

[21] J. Blazeck and H. S. Alper, "Promoter engineering: recent advances in controlling transcription at the most fundamental level," Biotechnology Journal, vol. 8, no. 1, pp. 46-58, 2013.

[22] C. Chai, Y. Lin, D. Shen, Y. Wu, H. Li, and D. Dou, "Identification and functional characterization of the soybean GmaPPO12 promoter conferring Phytophthora sojae induced expression," PLoS One, vol. 8, article e67670, 2013.

[23] H. Yoshioka, S. Asai, M. Yoshioka, and M. Kobayashi, "Molecular mechanisms of generation for nitric oxide and reactive oxygen species, and role of the radical burst in plant immunity," Molecules and Cells, vol. 28, pp. 321-329, 2009.

[24] L. Belbahri, C. Boucher, T. Candresse, M. Nicole, P. Ricci, and H. Keller, "A local accumulation of the Ralstonia solanacearum PopA protein in transgenic tobacco renders a compatible plant-pathogen interaction incompatible," The Plant Journal, vol. 28, pp. 419-430, 2001.

[25] H. Keller, N. Pamboukdjian, M. Ponchet et al., "Pathogeninduced elicitin production in transgenic tobacco generates a hypersensitive response and nonspecific disease resistance," The Plant Cell, vol. 11, pp. 223-235, 1999.

[26] H. Zhang, Y. R. Wang, and J. Lu, "Function and evolution of upstream ORFs in eukaryotes," Trends in Biochemical Sciences, vol. 44, pp. 782-794, 2019.

[27] T. Zhang, A. Wu, Y. Yue, and Y. Zhao, "uORFs: important CisRegulatory elements in plants," International Journal of Molecular Sciences, vol. 21, no. 17, 2020.

[28] M. J. Liu, S. H. Wu, J. F. Wu et al., "Translational landscape of photomorphogenic Arabidopsis," The Plant Cell, vol. 25, no. 10, pp. 3699-3710, 2013.

[29] H. Zhang, Y. R. Wang, X. K. Wu, X. L. Tang, C. C. Wu, and J. Lu, "Author Correction: Determinants of genome-wide distribution and evolution of uORFs in eukaryotes," Nature Communications, vol. 12, no. 1, 2021.

[30] R. X. Niu, Y. L. Zhou, Y. Zhang et al., "uORFlight: a vehicle toward uORF-mediated translational regulation mechanisms in eukaryotes," Database, vol. 2020, 2020.

[31] K. M. Pajerowska-Mukhtar, W. Wang, Y. Tada et al., "The HSF-like transcription factor TBF1 is a major molecular switch for plant growth-to-defense transition," Current Biology, vol. 22, pp. 103-112, 2012.

[32] N. Sotta, Y. Chiba, K. Miwa et al., "Global analysis of boroninduced ribosome stalling reveals its effects on translation termination and unique regulation by AUG-stops in Arabidopsis shoots," The Plant Journal, vol. 106, pp. 1455-1467, 2021.

[33] R. S. Reis, J. Deforges, T. Sokoloff, and Y. Poirier, "Modulation of shoot phosphate level and growth by PHOSPHATE1 upstream open reading frame," Plant Physiology, vol. 183, pp. 1145-1156, 2020.
[34] G. Xu, M. Yuan, C. Ai et al., "uORF-mediated translation allows engineered plant disease resistance without fitness costs," Nature, vol. 545, no. 7655, pp. 491-494, 2017.

[35] P. Brodersen, M. Petersen, H. M. Pike et al., "Knockout of Arabidopsis accelerated-cell-death11 encoding a sphingosine transfer protein causes activation of programmed cell death and defense," Genes \& Development, vol. 16, pp. 490-502, 2002.

[36] Q. Li, G. Ai, D. Shen et al., "A Phytophthora capsici effector targets ACD11 binding partners that regulate ROS-mediated defense response in Arabidopsis," Molecular Plant, vol. 12, pp. 565-581, 2019.

[37] H. Liu, S. Ravichandran, O. K. Teh et al., "The RING-type E3 ligase XBAT35.2 is involved in cell death induction and pathogen response," Plant Physiology, vol. 175, pp. 1469-1483, 2017.

[38] K. Bouwmeester, M. de Sain, R. Weide et al., "The lectin receptor kinase LecRK-I.9 is a novel Phytophthora resistance component and a potential host target for a RXLR effector," PLoS Pathogens, vol. 7, no. 3, p. e1001327, 2011.

[39] P. Singh and L. Zimmerli, "Lectin receptor kinases in plant innate immunity," Frontiers in Plant Science, vol. 4, p. 124, 2013.

[40] P. Singh, Y. C. Kuo, S. Mishra et al., "The lectin receptor kinase-VI.2 is required for priming and positively regulates Arabidopsis pattern-triggered immunity," The Plant Cell, vol. 24, pp. 1256-1270, 2012.

[41] C. Wang, X. Huang, Q. Li, Y. Zhang, J.-L. Li, and Z. Mou, "Extracellular pyridine nucleotides trigger plant systemic immunity through a lectin receptor kinase/BAK1 complex," Nature Communications, vol. 10, no. 1, p. 4810, 2019.

[42] P. Y. Huang, Y. H. Yeh, A. C. Liu, C. P. Cheng, and L. Zimmerli, "The Arabidopsis LecRK-VI.2 associates with the pattern-recognition receptor FLS2 and primes Nicotiana benthamiana pattern-triggered immunity," The Plant Journal, vol. 79, pp. 243-255, 2014.

[43] N. A. Rajput, M. Zhang, Y. Ru et al., "Phytophthora sojae effector PsCRN70 suppresses plant defenses in Nicotiana benthamiana," PLoS One, vol. 9, article e98114, 2014.

[44] G. Ai, Q. Xia, T. Song et al., "A Phytophthora sojae CRN effector mediates phosphorylation and degradation of plant aquaporin proteins to suppress host immune signaling," PLoS Pathogens, vol. 17, article e1009388, 2021.

[45] M. Tanaka, N. Sotta, Y. Yamazumi et al., "The minimum open reading frame, AUG-stop, induces boron-dependent ribosome stalling and mRNA degradation," The Plant Cell, vol. 28, pp. 2830-2849, 2016.

[46] H. McLellan, P. C. Boevink, M. R. Armstrong et al., "An RxLR effector from Phytophthora infestans prevents re-localisation of two plant NAC transcription factors from the endoplasmic reticulum to the nucleus," PLoS Pathogens, vol. 9, article e1003670, 2013.

[47] K. Goslin, L. Eschen-Lippold, C. Naumann et al., "Differential $\mathrm{N}$-end rule degradation of RIN4/NOI fragments generated by the AvrRpt2 effector protease," Plant Physiology, vol. 180, pp. 2272-2289, 2019.

[48] C. Mazo-Molina, S. Mainiero, B. J. Haefner et al., "Ptr1 evolved convergently with RPS2 and Mr5 to mediate recognition of AvrRpt2 in diverse solanaceous species," The Plant Journal, vol. 103, pp. 1433-1445, 2020.

[49] S. J. Park, K. Jiang, L. Tal et al., "Optimization of crop productivity in tomato using induced mutations in the florigen pathway," Nature Genetics, vol. 46, pp. 1337-1342, 2014. 
[50] D. Rodriguez-Leal, Z. H. Lemmon, J. Man, M. E. Bartlett, and Z. B. Lippman, "Engineering quantitative trait variation for crop improvement by genome editing," Cell, vol. 171, pp. 470-480 e478, 2017.

[51] Y. Eshed and Z. B. Lippman, "Revolutions in agriculture chart a course for targeted breeding of old and new crops," Science, vol. 366, no. 6466, p. 705, 2019.

[52] R. S. Meyer and M. D. Purugganan, "Evolution of crop species: genetics of domestication and diversification," Nature Reviews. Genetics, vol. 14, pp. 840-852, 2013.

[53] S. K. Thalor, T. Berberich, S. S. Lee et al., "Deregulation of sucrose-controlled translation of a bZIP-type transcription factor results in sucrose accumulation in leaves," PLoS One, vol. 7, 2012.

[54] G. L. Chew, A. Pauli, and A. F. Schier, "Conservation of uORF repressiveness and sequence features in mouse, human and zebrafish," Nature Communications, vol. 7, p. 11663, 2016.

[55] H. Zhang, X. Si, X. Ji et al., "Genome editing of upstream open reading frames enables translational control in plants," Nature Biotechnology, vol. 36, pp. 894-898, 2018.

[56] S. Katiyar-Agarwal, R. Morgan, D. Dahlbeck et al., "A pathogen-inducible endogenous siRNA in plant immunity," Proceedings of the National Academy of Sciences of the United States of America, vol. 103, pp. 18002-18007, 2006.

[57] H. Liu, Y. Li, Y. Hu et al., "EDS1-interacting J protein 1 is an essential negative regulator of plant innate immunity in Arabidopsis," The Plant Cell, vol. 33, pp. 153-171, 2021.

[58] G. Xu, G. H. Greene, H. Yoo et al., "Global translational reprogramming is a fundamental layer of immune regulation in plants," Nature, vol. 545, pp. 487-490, 2017.

[59] H. Yoo, G. H. Greene, M. Yuan et al., "Translational regulation of metabolic dynamics during effector-triggered immunity," Molecular Plant, vol. 13, pp. 88-98, 2020. 